\title{
A general method for studying quadratic perturbations of the third-order Lyness difference equation
}

\author{
Guifeng Deng ${ }^{1 *}$, Qiuying Lu² and Nianzu Liu'
}

\section{"Correspondence:}

maximedgf@163.com

'School of Mathematics and

Information Science, Shanghai Lixin

University of Commerce, Shanghai, 201620, P.R. China

Full list of author information is

available at the end of the article

\begin{abstract}
This paper studies the difference equation $x_{n+3} x_{n}=a+x_{n+1}+x_{n+2}+\gamma x_{n}^{2}$, where $a$ and $\gamma$ are arbitrary positive real numbers and the initial values $x_{0}, x_{1}, x_{2}>0$. It is known that for $\gamma=0$ the above equation is the third-order Lyness' one, studied in several papers. Using an extension of the quasi-Lyapunov method, we prove that for $0<\gamma<1$ the sequences generated by the perturbed third-order Lyness equation are globally asymptotically stable. Moreover, we show that if $\gamma \geq 1$ all solutions of it converge to $+\infty$. Therefore, the values 0 and 1 are two bifurcation points for the equation containing the parameter $\gamma$.
\end{abstract}

MSC: 39A 11;39A20

Keywords: difference equation; quadratic perturbations; bifurcation point; first integral; Lyapunov function; global asymptotic stability

\section{Introduction}

Lyness [1-3] discovered that the solutions of the second-order difference equation $x_{n+2} x_{n}=x_{n+1}+a$ is 5 -periodic for $a=1$ and positive initial conditions while he was working on a problem in Number Theory. The third-order Lyness equation is

$$
x_{n+3} x_{n}=a+x_{n+1}+x_{n+2}, \quad a>0, x_{0}, x_{1}, x_{2}>0 .
$$

According to Lyness [1-3], Todd has discovered every solution of equation (1) is 8-periodic if $a=1$. So, this equation is also known as Todd's equation. First Zeeman [4] and, after and independently, Bastien et al. [5] gave a complete description of global dynamics of the second-order Lyness equation with $a>0$ by the interpretation of the iteration of the map $F$ induced by this recurrence on the Lyness' cubic which passes through the initial points $\left(x_{1}, x_{0}\right)$. Cima et al. [6] applied an approach different to the ones in Refs. [5, 7] to study equation (1). That is, their main tool is the study of an ordinary differential equation associated to equation (1). They proved that for each $a \neq 1$ the periods of the sequences generated by equation (1) can be almost all natural numbers, depending on the initial points $\left(x_{0}, x_{1}\right)$. In recent years various generalized Lyness difference equations, including the Lyness difference equation with variable coefficients, the order $k$ Lyness difference equation and the perturbed Lyness-type order $k$ difference equation, have been extensively studied [8-14]. It is well known that there are no convergent nontrivial solutions for the Lyness difference

(c) 2013 Deng et al: licensee Springer. This is an Open Access article distributed under the terms of the Creative Commons Attribution License (http://creativecommons.org/licenses/by/2.0), which permits unrestricted use, distribution, and reproduction in any medium, provided the original work is properly cited. 
equation. In [8], Li found the convergent solutions of the Lyness difference equation with variable coefficients

$$
x_{n+2}=\frac{a_{n}+x_{n+1}}{x_{n}}, \quad x_{1}, x_{0}>0
$$

where $\left\{a_{n}\right\}$ is a monotonic non-increasing positive sequence, which demonstrates the essential difference between Lyness difference equation with constant coefficients and Lyness difference equation with variable coefficients.

In this paper we study the global asymptotic behavior of all solutions of the perturbed third-order Lyness difference equation

$$
x_{n+3}=\frac{a+x_{n+1}+x_{n+2}+\gamma x_{n}^{2}}{x_{n}}, \quad n=0,1, \ldots
$$

where $a, \gamma \in(0,+\infty)$ and the initial values $x_{0}, x_{1}, x_{2} \in(0,+\infty)$. It is clear that the quadratic term $x_{n}^{2}$ is a small perturbation for small positive number $\gamma$. The invariant curve of difference equations often plays a critical role in studying the stability behavior of their solutions; see [5, 15, 16]. Kocic et al. [17] have applied KAM theory to prove the stability of the solutions of the Lyness equation. Meanwhile, Lyapunov functions have been found in this area by several papers; see $[12,15,16]$. Here, using an extension of the method introduced in [12], which itself generalizes an idea of Merino [18], we obtain the preservation of global asymptotic stability for the third-order Lyness difference equation under quadratic perturbations.

Our main result is the following.

Theorem 1.1 (1) If $0<\gamma<1$, then the positive equilibrium point $l_{\gamma}$ of equation (2) is globally asymptotically stable, where

$$
l_{\gamma}=\frac{1+\sqrt{1+a(1-\gamma)}}{1-\gamma} .
$$

(2) If $\gamma \geq 1$, then the sequence $\left\{x_{n}\right\}_{n=0}^{\infty}$ generated by equation (2) converges to $+\infty$.

Cima et al. [6] proved that for a given $a>0$ there exist periodic sequences $\left\{x_{n}\right\}_{n=0}^{\infty}$ generated by equation (1) which have almost all long periods and that for a full measure set of initial conditions the sequences $\left\{x_{n}\right\}_{n=0}^{\infty}$ are dense in either one or two disjoint bounded intervals of $R$. In summary, the sequences $\left\{x_{n}\right\}_{n=0}^{\infty}$ are periodic or strictly oscillatory, and the equilibrium point $l_{0}$ is locally stable, with $l_{0}=1+\sqrt{1+a}$. That is, for every $\epsilon>0$, there is a $\delta>0$ so that, for any positive initial values $x_{0}, x_{1}$ and $x_{2}$ with $\left|x_{i}-l_{0}\right|<\delta$ for $i=0,1,2$, one has $\left|x_{n}-l_{0}\right|<\epsilon$ for all $n \geq 0$. According to Theorem 1.1, the sequences $\left\{x_{n}\right\}_{n=0}^{\infty}$ of solutions of equation (2) are converging to $l_{\gamma}$ if $0<\gamma<1$. So, the qualitative nature of the solutions of equation (2) changes when $\gamma$ vanishes. Note that the sequences $\left\{x_{n}\right\}_{n=0}^{\infty}$ are increasing to $+\infty$ if $\gamma \geq 1$. So, the behavior of the solutions of equation (2) is completely different from the case $0<\gamma<1$. Thus, the values 0 and 1 are two bifurcation points for the difference equation (2) containing the parameter $\gamma$. 


\section{Lyapunov function}

In this section we assume that $0<\gamma<1$. We begin by introducing the nonlinear map

$$
F(x, y, z)=\left(y, z, \frac{a+y+z+\gamma x^{2}}{x}\right)
$$

Let $\left\{x_{n}\right\}_{n=0}^{\infty}$ be the sequence generated by equation (2) with positive initial conditions, then it is not difficult to check that $F\left(x_{n}, x_{n+1}, x_{n+2}\right)=\left(x_{n+1}, x_{n+2}, x_{n+3}\right)$ for all $n \geq 0$. Moreover, $\left(l_{\gamma}, l_{\gamma}, l_{\gamma}\right)$ is a unique fixed point of $F$ in $R_{+}^{3}$, where $R_{+}^{3}=\{(x, y, z) \mid x>0, y>0, z>0\}$ and $l_{\gamma}=\frac{1+\sqrt{1+a(1-\gamma)}}{1-\gamma}$. It is well known $[6,15]$ that for $\gamma=0$ equation (2) possesses the following first integral:

$$
V(x, y, z)=\frac{(x+1)(y+1)(z+1)(a+x+y+z)}{x y z} .
$$

In other words, $F$ maps the level surface $V^{-1}(k)$ into itself for every k when $\gamma=0$. In order to study the global convergence properties of the recurrence (2) for $0<\gamma<1$, we introduce a very important function $I(x, y, z)$ given by

$$
I(x, y, z)=\frac{[(1-\gamma) x+1][(1-\gamma) y+1][(1-\gamma) z+1](a+x+y+z)}{(1-\gamma)^{2} x y z} .
$$

In fact, the function $I(x, y, z)$ is the same as $V(x, y, z)$ for $\gamma=0$. Using the function $I$, we construct the Lyapunov function $L(x, y, z)$ of equation (2). Set $k_{\gamma}=I\left(l_{\gamma}, l_{\gamma}, l_{\gamma}\right)$, then we show that the Lyapunov function is

$$
L(x, y, z)=I(x, y, z)-I\left(l_{\gamma}, l_{\gamma}, l_{\gamma}\right)=I(x, y, z)-k_{\gamma} .
$$

Let us determine some properties of the function $I(x, y, z)$. Note that if $0 \leq \gamma<1$, we get the relations

$$
\begin{aligned}
& \lim _{x \rightarrow 0^{+}} I(x, y, z)=\lim _{y \rightarrow 0^{+}} I(x, y, z)=\lim _{z \rightarrow 0^{+}} I(x, y, z)=+\infty, \\
& \lim _{x^{2}+y^{2} \rightarrow+\infty} I(x, y, z)=\lim _{x^{2}+z^{2} \rightarrow+\infty} I(x, y, z)=\lim _{y^{2}+z^{2} \rightarrow+\infty} I(x, y, z)=+\infty
\end{aligned}
$$

and

$$
\lim _{x^{2}+y^{2}+z^{2} \rightarrow+\infty} I(x, y, z)=+\infty
$$

It is easy to see that the function $I(x, y, z)$ has continuous second partial derivatives in $R_{+}^{3}$. Thus, the possible critical points are the stationary points obtained by setting $I_{x}(x, y, z)$, $I_{y}(x, y, z)$ and $I_{z}(x, y, z)$ equal to zero. Taking partial derivatives and setting them equal to 0 gives

$$
\begin{aligned}
& I_{x}(x, y, z)=\frac{[(1-\gamma) y+1][(1-\gamma) z+1]\left[(1-\gamma) x^{2}-a-y-z\right]}{(1-\gamma)^{2} x^{2} y z}=0, \\
& I_{y}(x, y, z)=\frac{[(1-\gamma) x+1][(1-\gamma) z+1]\left[(1-\gamma) y^{2}-a-x-z\right]}{(1-\gamma)^{2} x y^{2} z}=0,
\end{aligned}
$$




$$
I_{z}(x, y, z)=\frac{[(1-\gamma) x+1][(1-\gamma) y+1]\left[(1-\gamma) z^{2}-a-x-y\right]}{(1-\gamma)^{2} x y z^{2}}=0 .
$$

The unique positive solution to this system of equations is

$$
x_{c}=y_{c}=z_{c}=l_{\gamma}=\frac{1+\sqrt{1+a(1-\gamma)}}{1-\gamma} .
$$

Next, we check the Hessian $H$ at the stationary point $P_{\gamma}=\left(x_{c}, y_{c}, z_{c}\right)=\left(l_{\gamma}, l_{\gamma}, l_{\gamma}\right)$, where

$$
H=\left(\begin{array}{lll}
A & B & B \\
B & A & B \\
B & B & A
\end{array}\right) .
$$

Computing the second-order derivatives of $I$, we get

$$
A=\left.\frac{\partial^{2} I}{\partial^{2} x}\right|_{P_{\gamma}}=\left.\frac{\partial^{2} I}{\partial^{2} y}\right|_{P_{\gamma}}=\left.\frac{\partial^{2} I}{\partial^{2} z}\right|_{P_{\gamma}}=\frac{2\left[(1-\gamma) l_{\gamma}+1\right]^{2}}{(1-\gamma) l_{\gamma}^{3}}>0
$$

and

$$
B=\left.\frac{\partial^{2} I}{\partial x \partial y}\right|_{P_{\gamma}}=\left.\frac{\partial^{2} I}{\partial x \partial z}\right|_{P_{\gamma}}=\left.\frac{\partial^{2} I}{\partial y \partial z}\right|_{P_{\gamma}}=\frac{\left[(1-\gamma) l_{\gamma}+1\right]\left[a+l_{\gamma}-2(1-\gamma) l_{\gamma}^{2}\right]}{(1-\gamma)^{2} l_{\gamma}^{5}}
$$

Let $H_{m}$ be the $m$ th-order principal minor of the Hessian $H$. Then we obtain

$$
\operatorname{det} H_{1}=A>0, \quad \operatorname{det} H_{2}=\frac{\left[(1-\gamma) l_{\gamma}+1\right]^{4}\left[4(1-\gamma)^{2} l_{\gamma}^{2}-1\right]}{(1-\gamma)^{4} l_{\gamma}^{8}}>0,
$$

and

$$
\operatorname{det} H=\frac{2\left[(1-\gamma) l_{\gamma}+1\right]^{6}\left[2(1-\gamma) l_{\gamma}+1\right]^{2}\left[(1-\gamma) l_{\gamma}-1\right]}{(1-\gamma)^{6} l_{\gamma}^{12}}>0 .
$$

Thus, the function $I(x, y, z)$ attains a strict minimum at $\left(l_{\gamma}, l_{\gamma}, l_{\gamma}\right)$ in $R_{+}^{3}$, and it has no other critical point. We obtain

$$
k_{\gamma}=I\left(l_{\gamma}, l_{\gamma}, l_{\gamma}\right)=\frac{(\sqrt{(1-\gamma) a+1}+2)^{3}[(1-\gamma) a+3+3 \sqrt{(1-\gamma) a+1}]}{[\sqrt{(1-\gamma) a+1}+1]^{3}} .
$$

Let $I_{k}=\left\{(x, y, z) \mid I(x, y, z)=k,(x, y, z) \in R_{+}^{3}\right\}$ be the level surface of $I$ for $k>k_{\gamma}$. It is well known that the function $V(x, y, z)$ has a minimum at $(\tilde{x}, \tilde{x}, \tilde{x})$ in $R_{+}^{3}$, where $\tilde{x}=1+\sqrt{1+a}$. In order to study the third-order Lyness equation, Cima et al. [6] proved the following result.

Lemma 2.1 Let $L_{k}=\left\{(x, y, z) \mid V(x, y, z)=k,(x, y, z) \in R_{+}^{3}\right\}$, and put $k_{c}=V(\tilde{x}, \tilde{x}, \tilde{x})$. Then set $L_{k}$ is diffeomorphic to a sphere $\Omega^{2}$ for $k>k_{c}$.

To describe the level surface $I(x, y, z)=k$ for $k>k_{\gamma}$, we apply the invertible linear transformation of variables

$$
(u, v, w)=\varphi(x, y, z)=((1-\gamma) x,(1-\gamma) y,(1-\gamma) z),
$$


which induces an isomorphism between the set $I_{k}$ and the level surface $\widetilde{L}_{k}=\{(u, v, w) \mid$ $\left.V_{a(1-\gamma)}(u, v, w)=k,(u, v, w) \in R_{+}^{3}\right\}$, where

$$
V_{a(1-\gamma)}(u, v, w)=\frac{(u+1)(v+1)(w+1)[a(1-\gamma)+u+v+w]}{u v w} .
$$

By using Lemma 2.1, we have that $\widetilde{L}_{k}$ is diffeomorphic to a sphere for $0<\gamma<1$. This means that $I_{k}$ is also diffeomorphic to a sphere for $k>k_{\gamma}$. So, we proved the corollary as follows.

Proposition 2.2 For $k>k_{\gamma}$ the level surface $I_{k}$ is diffeomorphic to a sphere.

It is easy to see that the set $I_{k}$ is compact for $k>k_{\gamma}$. Moreover, Cima et al. [6] introduced an important curve $\mathcal{L}$ that is an invariant, where

$$
\mathcal{L}=\left\{\left(t, \frac{t+a}{t-1}, t\right) \mid t>1\right\}
$$

Here, according to the map $F$, we define a curve $\widetilde{\mathcal{L}}$ given by

$$
\widetilde{\mathcal{L}}:=\left\{\left(s, \frac{s+a}{(1-\gamma) s-1}, s\right) \mid s>\frac{1}{1-\gamma}\right\} \subset R_{+}^{3} .
$$

It is easy to check that $\widetilde{\mathcal{L}}$ contains the unique fixed point $\left(l_{\gamma}, l_{\gamma}, l_{\gamma}\right)$. Let

$$
h(s)=\left.I\right|_{\widetilde{\mathcal{L}}}=I\left(s, \frac{s+a}{(1-\gamma) s-1}, s\right)=\frac{[(1-\gamma) s+1]^{2}[(1-\gamma)(2 s+a)-1]^{2}}{(1-\gamma)^{2} s(s+a)[(1-\gamma) s-1]}, \quad s>\frac{1}{1-\gamma} .
$$

A straightforward calculation shows that $l_{\gamma}$ is the unique solution of $h^{\prime}(s)=0$ and that $\lim _{(1-\gamma) s \rightarrow 1^{+}} h(s)=\lim _{s \rightarrow+\infty} h(s)=+\infty$. Hence, $I_{k} \cap \widetilde{\mathcal{L}}$ consists of two points for every $k>k_{\gamma}$. It is easy to show that the set $D_{k}=\left\{(x, y, z) \mid I(x, y, z) \leq k,(x, y, z) \in R_{+}^{3}\right\}$ contains the unique critical point $\left(l_{\gamma}, l_{\gamma}, l_{\gamma}\right)$ for $k>k_{\gamma}$. Moreover, $D_{k}$ is compact with $k>k_{\gamma}$.

Remark 2.3 According to the transformation (5), we have $I(x, y, z)=V_{a(1-\gamma)}(u, v, w)$. In [13], the authors obtained a classical property of the function $V_{a(1-\gamma)}(u, v, w)$ : it tends to $+\infty$ at the infinity point of the locally compact space $\{(u, v, w) \mid u>0, v>0, w>0\}$. Then the property of $I(x, y, z)$ is the same as this one of $V_{a(1-\gamma)}(u, v, w)$. This is a direct proof about the property of the set $D_{k}$ for $k>k_{\gamma}$. Furthermore, the sets $D_{k}$ for $k>k_{\gamma}$ form a system of compact neighborhood of the fixed point $\left(l_{\gamma}, l_{\gamma}, l_{\gamma}\right)$.

In order to simplify the proof of our main result, we need the following result.

Proposition 2.4 Set $S=\left\{(x, y, z) \mid(1-\gamma) x^{2}=a+y+z,(x, y, z) \in R_{+}^{3}\right\}$ and $\Gamma=\{(x, y, z) \mid(1-$ $\left.\gamma) x^{2}=a+y+z,(1-\gamma) y^{2}=a+x+z,(x, y, z) \in R_{+}^{3}\right\}$. If $0<\gamma<1$, then

(1) $I(F(x, y, z))<I(x, y, z)$ for all points $(x, y, z) \in R_{+}^{3} \backslash S$, and $I(F(x, y, z))=I(x, y, z)$ for $(x, y, z) \in S$.

(2) $I\left(F^{2}(x, y, z)\right)<I(x, y, z)$ for every point $(x, y, z) \in S \backslash \Gamma$, and $I\left(F^{2}(x, y, z)\right)=I(x, y, z)$ for $(x, y, z) \in \Gamma$.

(3) $I\left(F^{3}(x, y, z)\right)<I(x, y, z)$ for all $(x, y, z) \in \Gamma$ except the fixed point $\left(l_{\gamma}, l_{\gamma}, l_{\gamma}\right)$. 
Proof Note that $\gamma$ satisfies the relation $0<\gamma<1$. Using (3) and (4), we have

$$
\begin{aligned}
L(x, y, z)-L(F(x, y, z)) & \\
= & I(x, y, z)-I(F(x, y, z)) \\
= & \frac{[(1-\gamma) y+1][(1-\gamma) z+1]}{(1-\gamma)^{2} y z} \\
& \times\left\{\frac{[(1-\gamma) x+1](a+x+y+z)}{x}-\frac{\left[(1-\gamma) \frac{a+y+z+\gamma x^{2}}{x}+1\right]\left(a+y+z+\frac{a+y+z+\gamma x^{2}}{x}\right)}{\frac{a+y+z+\gamma x^{2}}{x}}\right\} \\
= & -\frac{[(1-\gamma) y+1][(1-\gamma) z+1]}{(1-\gamma)^{2} y z\left(a+y+z+\gamma x^{2}\right)} \\
& \times\left\{(1-\gamma) x\left(\frac{a+y+z+\gamma x^{2}}{x}\right)\right)^{2} \\
& \left.-\left[a+y+z+(1-\gamma) x^{2}\right] \frac{a+y+z+\gamma x^{2}}{x}+x(a+y+z)\right\} \\
= & \frac{[(1-\gamma) y+1][(1-\gamma) z+1]}{(1-\gamma)^{2} x y z} \frac{x}{a+y+z+\gamma x^{2}}(1-\gamma) \\
= & \frac{x\left[\frac{a+y+z+\gamma x^{2}}{x}-\frac{a+y+z}{(1-\gamma) x}\right]\left(x-\frac{a+y+z+\gamma x^{2}}{x}\right)}{x+y+z+\gamma x^{2}} \frac{[(1-\gamma) y+1][(1-\gamma) z+1] \gamma}{(1-\gamma)^{2} y z}\left[(1-\gamma) x-\frac{a+y+z}{x}\right]^{2} \\
& \times x
\end{aligned}
$$

Therefore, from the above expression, we obtained the assertion (1).

For every point $(x, y, z)$ belonging to $S$, then the equality $(1-\gamma) x^{2}=a+y+z$ is fulfilled. From this, we conclude that

$$
F(F(x, y, z))=F\left(y, z, \frac{a+y+z+\gamma x^{2}}{x}\right)=F(y, z, x)=\left(z, x, \frac{a+z+x+\gamma y^{2}}{y}\right) .
$$

Thus,

$$
\begin{aligned}
L(x, y, z)-L(F(F(x, y, z))) & \\
= & I(x, y, z)-I\left(z, x, \frac{a+z+x+\gamma y^{2}}{y}\right) \\
= & \frac{[(1-\gamma) z+1][(1-\gamma) x+1]}{(1-\gamma)^{2} x z} \\
& \times\left\{\frac{[(1-\gamma) y+1](a+x+y+z)}{y}-\frac{\left[(1-\gamma) \frac{a+z+x+\gamma y^{2}}{y}+1\right]\left(a+x+z+\frac{a+z+x+\gamma y^{2}}{y}\right)}{\frac{a+z+x+\gamma y^{2}}{y}}\right\} \\
= & \frac{[(1-\gamma) x+1][(1-\gamma) z+1] \gamma}{a+x+z+\gamma y^{2}}\left[(1-\gamma) y-\frac{a+x+z}{y}\right]^{2} \geq 0 .
\end{aligned}
$$

If $0<\gamma<1$, then the expression $I(x, y, z)-I(F(F(x, y, z)))$ equals zero if and only if $a+x+z=$ $(1-\gamma) y^{2}$. Note that the coordinates of the point $(x, y, z)$ satisfy the equality $a+y+z=$ $(1-\gamma) x^{2}$. From these equations, we proved the assertion (2). 
Suppose that $(x, y, z) \in \Gamma$, then it is easy to check that

$$
F^{3}(x, y, z)=F^{2}(y, z, x)=F\left(z, x, \frac{a+z+x+\gamma y^{2}}{y}\right)=F(z, x, y)=\left(x, y, \frac{a+x+y+\gamma z^{2}}{z}\right) .
$$

Therefore,

$$
\begin{aligned}
& L(x, y, z)-L\left(F^{3}(x, y, z)\right) \\
& =I(x, y, z)-I\left(x, y, \frac{a+x+y+\gamma z^{2}}{z}\right) \\
& =\frac{[(1-\gamma) x+1][(1-\gamma) y+1]}{(1-\gamma)^{2} x y} \\
& \quad \times\left\{\frac{[(1-\gamma) z+1](a+x+y+z)}{z}-\frac{\left[(1-\gamma) \frac{a+x+y+\gamma z^{2}}{z}+1\right]\left(a+x+y+\frac{a+x+y+\gamma z^{2}}{z}\right)}{\frac{a+x+y+\gamma z^{2}}{z}}\right\} \\
& =\frac{[(1-\gamma) x+1][(1-\gamma) y+1] \gamma}{a+x+y+\gamma z^{2}}\left[(1-\gamma) z-\frac{a+x+y}{z}\right]^{2} \geq 0 .
\end{aligned}
$$

Obviously, the expression $I(x, y, z)-I\left(F^{3}(x, y, z)\right)$ equals zero if and only if $a+x+y=(1-\gamma) z^{2}$ for $0<\gamma<1$. Note that the point $(x, y, z)$ belongs to $\Gamma$. It is easy to see that there exists a unique positive solution $(x, y, z)=\left(l_{\gamma}, l_{\gamma}, l_{\gamma}\right)$ such that $I(x, y, z)=I\left(F^{3}(x, y, z)\right)$ (see first from the three equations $(1-\gamma) x^{2}=a+y+z,(1-\gamma) y^{2}=a+x+z$ and $(1-\gamma) z^{2}=a+x+y$ that $x=y=z$ ). Then we proved the assertion (3).

Remark 2.5 It is clear that the inequality $L(F(x, y, z)) \leq L(x, y, z)$ holds for $0<\gamma<1$. Then $L(x, y, z)$ is called a Lyapunov function [16]. In summary, we deduce that $I\left(F^{3}(x, y, z)\right)<$ $I(x, y, z)$ holds for $(x, y, z) \in R_{+}^{3}$ except the unique fixed point $\left(l_{\gamma}, l_{\gamma}, l_{\gamma}\right)$. Let the map $F$ in the case $\gamma=0$ be denoted by $\left.F\right|_{\gamma=0}$. The level surface $V(x, y, z)=k$ is an invariant $[15,16]$ for $\left.F\right|_{\gamma=0}$. We also call the function $V(x, y, z)$ the first integral of the map $\left.F\right|_{\gamma=0}$. Using the function $V(x, y, z)$, we obtain the Lyapunov function $I(x, y, z)$ for equation (2).

\section{Proof of Theorem 1.1}

In this section we prove the main result.

Proof Now, we show that the positive equilibrium point $l_{\gamma}$ of equation (2) is globally asymptotically stable for $0<\gamma<1$.

Recall that $k_{\gamma}=I\left(l_{\gamma}, l_{\gamma}, l_{\gamma}\right)$ is a global minimum value for the function $I(x, y, z)$ in (4). By Proposition 2.2, the level surface $I(x, y, z)=k$ is diffeomorphic to a sphere for every $k>k_{\gamma}$. Moreover, the level surface $I(x, y, z)=k$ continuously shrinks to a point $\left(l_{\gamma}, l_{\gamma}, l_{\gamma}\right)$ as $k \rightarrow k_{\gamma}^{+}$. Note that the equality $I\left(x_{3 n}, x_{3 n+1}, x_{3 n+2}\right)=I\left(F^{3 n}\left(x_{0}, x_{1}, x_{2}\right)\right)$ is fulfilled for all positive initial conditions, where the sequence $\left\{x_{n}\right\}_{n=0}^{\infty}$ is the solution of equation (2). It follows from Proposition 2.4 that for $(x, y, z) \neq\left(l_{\gamma}, l_{\gamma}, l_{\gamma}\right)$ the monotone sequence $\left\{I\left(F^{3 n}(x, y, z)\right)\right\}_{n=0}^{\infty}$ converges to $k_{\gamma}$ as $n \rightarrow \infty$. Otherwise, there must be a real number $k>k_{\gamma}$ such that $\lim _{n \rightarrow \infty} I\left(F^{3 n}\left(x_{0}, x_{1}, x_{2}\right)\right)=k$ for some $\left(x_{0}, x_{1}, x_{2}\right) \in R_{+}^{3}$. It is easy to see that the sequence $\left\{F^{3 n}\left(x_{0}, x_{1}, x_{2}\right)\right\}_{n=0}^{\infty}$ belongs to a set $D=\left\{(x, y, z) \mid k \leq I(x, y, z) \leq I\left(x_{0}, x_{1}, x_{2}\right),(x, y, z) \in R_{+}^{3}\right\}$. Obviously, the set $D$ is closed, bounded and compact. Since there must be a subsequence 
$\left\{F^{3 n_{l}}\left(x_{0}, x_{1}, x_{2}\right)\right\}_{l=0}^{\infty}$ such that $\lim _{l \rightarrow \infty} F^{3 n_{l}}\left(x_{0}, x_{1}, x_{2}\right)=(\widetilde{x}, \tilde{y}, \widetilde{z})$, where $(\widetilde{x}, \tilde{y}, \widetilde{z}) \in D$. Next, considering another subsequence $\left\{F^{3}\left(F^{3 n_{l}}\left(x_{0}, x_{1}, x_{2}\right)\right)\right\}_{l=0}^{\infty}$ of $\left\{F^{3 n}\left(x_{0}, x_{1}, x_{2}\right)\right\}_{n=0}^{\infty}$, we obtain that $\lim _{l \rightarrow \infty} I\left(F^{3}\left(F^{3 n_{l}}\left(x_{0}, x_{1}, x_{2}\right)\right)\right)=k$. Because $I(x, y, z)$ and $F(x, y, z)$ are continuous functions in $R_{+}^{3}$, it is not difficult to deduce that $I(\widetilde{x}, \widetilde{y}, \widetilde{z})=\lim _{l \rightarrow \infty} I\left(F^{3 n_{l}}\left(x_{0}, x_{1}, x_{2}\right)\right)=k$ and

$$
k=\lim _{l \rightarrow \infty} I\left(F^{3}\left(F^{3 n_{l}}\left(x_{0}, x_{1}, x_{2}\right)\right)\right)=I\left(F^{3} \lim _{l \rightarrow \infty}\left(F^{3 n_{l}}\left(x_{0}, x_{1}, x_{2}\right)\right)\right)=I\left(F^{3}(\widetilde{x}, \tilde{y}, \widetilde{z})\right)<I(\widetilde{x}, \tilde{y}, \widetilde{z}) .
$$

This is a contradiction. So, we have $\lim _{n \rightarrow \infty} I\left(F^{3 n}(x, y, z)\right)=k_{\gamma}$ for all $(x, y, z) \in R_{+}^{3}$. There-

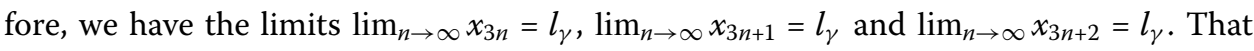
is, the positive equilibrium point $l_{\gamma}$ of equation (2) is globally asymptotically stable when $0<\gamma<1$.

Finally, we give the proof of the assertion (2) of Theorem 1.1. Note that the initial values $x_{0}, x_{1}$ and $x_{2}$ are positive real numbers, and the parameters $a$ and $\gamma$ are also positive in equation (2). For $\gamma \geq 1$, we have

$$
x_{n+3}=\frac{a+x_{n+1}+x_{n+2}+\gamma x_{n}^{2}}{x_{n}}>\frac{a}{x_{n}}+x_{n}>x_{n} .
$$

So, the subsequences $\left\{x_{3 n}\right\}_{n=0}^{\infty},\left\{x_{3 n+1}\right\}_{n=0}^{\infty}$ and $\left\{x_{3 n+2}\right\}_{n=0}^{\infty}$ of solution $\left\{x_{n}\right\}_{n=0}^{\infty}$ of equation (2) are monotonically increasing. Then the sequence $\left\{x_{n}\right\}_{n=0}^{\infty}$ converges to $+\infty$ as $n \rightarrow \infty$. Otherwise, there must be an integer $m$ such that $\lim _{n \rightarrow \infty} x_{3 n+m}=b$ for $0 \leq m \leq 2$. We write $3 n+m$ in place of $n$ in equation (6). This gives the recurrence relation

$$
x_{3 n+m+3}>\frac{a}{x_{3 n+m}}+x_{3 n+m}
$$

Take the limits on both sides of the above inequality and obtain $b \geq \frac{a}{b}+b$. This is a contradiction. Hence, the sequence $\left\{x_{n}\right\}_{n=0}^{\infty}$ converges to $+\infty$ as $n \rightarrow \infty$ if $\gamma \geq 1$. The proof is completed.

Remark 3.1 Indeed, using the extension of the quasi-Lyapounov method, we can obtain the global asymptotic behavior of the second-order Lyness equation $x_{n+2} x_{n}=a+x_{n+1}+\gamma x_{n}^{2}$ for certain domains of values for parameters.

\section{Competing interests \\ The authors declare that they have no competing interests.}

\section{Authors' contributions}

GD carried out the study of the third-order difference equation and drafted the manuscript. QL conceived of the study and helped to draft the manuscript. NL was involved in revising it critically for important intellectual content. All authors have read and approved the final manuscript.

\section{Author details}

${ }^{1}$ School of Mathematics and Information Science, Shanghai Lixin University of Commerce, Shanghai, 201620, P.R. China.

${ }^{2}$ Department of Mathematics, Zhejiang Sci-Tech University, Hangzhou, Zhejiang Province 310018, P.R. China.

\section{Acknowledgements}

We thank the anonymous referees for their careful reading of the manuscript and their helpful comments. This work was supported by the National Natural Science Foundation of China (No: 11101283, No: 11101370), Innovation Program of Shanghai Municipal Education Commission (No: 12YZ173), SRF for Rocs, SEM (No: 114329A4C11604), and the Group of Accounting and Governance Disciplines (No: 10kq03). 


\section{References}

1. Lyness, RC: Note 1581. Math. Gaz. 26, 62 (1942)

2. Lyness, RC: Note 1847. Math. Gaz. 29, 231 (1945)

3. Lyness, RC: Note 2952. Math. Gaz. 45, 201 (1961)

4. Zeeman, EC: Geometric unfolding of a difference equation. Unpublished paper. Hertford College, Oxford (1996)

5. Bastien, $G$, Rogalski, M: Global behaviour of the solutions of Lyness' difference equations $u_{n+2} u_{n}=u_{n+1}+a$. J. Differ. Equ. Appl. 10(11), 977-1003 (2004)

6. Cima, A, Gasull, A, Mañosa, V: Dynamics of the third order Lyness' difference equation. arXiv:math.DS/0612407

7. Beukers, F, Cushman, R: Zeeman's monotonicity conjecture. J. Differ. Equ. 143, 191-200 (1998)

8. Li, XY: A counterexample to Ladas' conjecture for Lyness equation. Chin. Sci. Bull. 43(16), 1788 (1998) (in Chinese)

9. Li, XY, Xiao, GF: Periodicity and strict oscillation for generalized Lyness equations. Appl. Math. Mech. 21(4), 455-460 (2000)

10. Cima, A, Gasull, A, Mañosa, V: On 2- and 3-periodic Lyness difference equations. J. Differ. Equ. Appl. 18(5), 849-864 (2012)

11. Feuer, J: Periodic solutions of the Lyness max equation. J. Math. Anal. Appl. 288(2), 147-160 (2003)

12. Bastien, $G$, Rogalski, M: Results and problems about solutions of perturbed Lyness' type order $k$ difference equations in $R_{*}^{+} u_{n+k}\left(u_{n}+\lambda\right)=f\left(u_{n+k-1}, \ldots, u_{n+1}\right)$, with examples, and test of the efficiency of a quasi-Lyapunov function method. J. Differ. Equ. Appl. (2012). doi:10.1080/10236198.2012.748758

13. Bastien, $G$, Rogalski, M: Results and conjectures about order q Lyness' difference equation $u_{n+k} u_{n}=a+u_{n+q-1}+\cdots+u_{n+1}$ in $R_{*}^{+}$, with a particular study of the case $q=3$. Adv. Differ. Equ. 2009, Article ID 134749 (2009)

14. Bastien, G, Rogalski, M: Global behaviour of solutions of cyclic systems of $q$ order 2 or 3 generalized Lyness' difference equations and of other more general equations of higher order. J. Differ. Equ. Appl. 17(11), 1651-1672 (2011)

15. Gao, M, Kato, Y: Some invariants for kth-order Lyness equation. Appl. Math. Lett. 17(10), 1183-1189 (2004)

16. Kulenović, MRS: Invariants and related Liapunov functions for difference equations. Appl. Math. Lett. 13(7), 1-8 (2000)

17. Kocic, VL, Ladas, G, Tzanetopoulos, G, Thomas, E: On the stability of Lyness' equation. Dyn. Contin. Discrete Impuls. Syst. 1, 245-254 (1995)

18. Merino, $\mathrm{O}$ : Global attractivity of the equilibrium of a difference equation: an elementary proof assisted by computer algebra system. J. Differ. Equ. Appl. 17(1), 34-41 (2011)

doi:10.1186/1687-1847-2013-193

Cite this article as: Deng et al.: A general method for studying quadratic perturbations of the third-order Lyness difference equation. Advances in Difference Equations 2013 2013:193.

\section{Submit your manuscript to a SpringerOpen ${ }^{\circ}$ journal and benefit from:}

- Convenient online submission

Rigorous peer review

- Immediate publication on acceptance

- Open access: articles freely available online

- High visibility within the field

- Retaining the copyright to your article 